\title{
The utilization of cytologic and small biopsy samples for ancillary molecular testing
}

\author{
Michael H. Roh ${ }^{1}$
}

Received: 20 July 2018 / Accepted: 22 July 2018 / Published online: 2 January 2019

(c) United States \& Canadian Academy of Pathology 2019

\begin{abstract}
There has recently been an increased emphasis on the utilization of cytologic samples and small biopsies for not only diagnostic purposes but also for ancillary testing. In some instances, the ancillary tests contribute to the diagnosis and in other scenarios, they provide prognostic and theranostic information for the management of patients with advanced stage cancer. These ancillary tests include immunohistochemical biomarker analysis, molecular mutation analysis, and cytogenetic tests. Despite the finite nature of the cellular material procured in cytologic and small tissue biopsies, pathologists are tasked with ordering an increasing number of tests using these limited samples. This requires the pathologists to utilize and triage these samples in an optimal fashion so that as much information can be gleaned from a given specimen. This review will focus on the pre-analytic requirements for ancillary molecular and cytogenetic tests in the context of a discussion of the various preparation methods for cytologic and small biopsy specimens. The goal will be to provide the reader with the necessary concepts that can be utilized to develop optimal specimen selection and triage strategies to maximize the chances of effectively utilizing these samples for comprehensive diagnostic and relevant ancillary testing purposes.
\end{abstract}

\section{Introduction}

The current era of precision medicine has been associated with rapid advances in our understanding of molecular drivers of various malignancies and our ability to test for and detect a variety of genetic abnormalities. Improvements in our ability to detect and sample primary malignancies and metastatic tumors, via minimally invasive interventional procedures, for pathologic evaluation have led to a greater emphasis on the role of cytologic and small biopsy samples in not only accurately diagnosing tumors but also in the molecular characterization of these cancers [1-3]. The volume of these cytologic samples, especially fine-needle aspirates (FNAs), and small biopsies are finite in quantity. Nonetheless, pathologists have witnessed an increasing diversification in which molecular diagnostic applications

Michael H. Roh

Roh.Michael@mayo.edu

1 Division of Anatomic Pathology, Department of Laboratory Medicine and Pathology, Mayo Clinic, 200 First Street SW, Rochester, MN 55905, United States have been applied to cytologic samples and small biopsies. Hence, pathologists are tasked with the challenge to obtain an increasing amount of information from these samples including accurate pathologic diagnoses and genetic/biomarker data via immunohistochemistry, molecular tests, and/or cytogenetic tests. These data include gene mutations, amplifications, and gene rearrangements to name a few examples.

In patients with advanced cancer, a small biopsy sample or cytologic sample may be the only opportunity to obtain tumor material for diagnosis and ancillary testing. Therefore, pathologists are at the forefront in managing these patient assets by reliably triaging these samples in a judicious, optimal fashion in order to meet these challenges [2]. Occasionally, repeat procedures are needed to collect additional cytologic and/or small biopsy samples from patients' cancers solely for the purpose of ancillary molecular, immunohistochemical, or cytogenetic testing. These repeat procedures are not without risk, however, and may not guarantee acquisition of sufficient quality material for these purposes. Furthermore, having to perform repeat biopsies also essentially introduces delays in patient management. Therefore, with each and every patient sample, based on the clinical context and the type of sample being 
collected, the pathologist should make the best effort to appropriately triage patient samples for diagnostically relevant tests as well as potential downstream ancillary tests that would stem from the pathologic diagnoses. This requires an understanding of sample requirements for these tests, the platforms (e.g., formalin fixed paraffin embedded blocks, direct smears, liquid based preparations) which have been validated for the tests, along with strengths and limitations of these platforms for given testing methodologies. Understandably, the degrees to which platforms and ancillary tests have been validated are institution specific. Pathologists, armed with the principles discussed herein, should be in a position to devise best practice procedures in collaboration with their clinical colleagues at their respective institutions.

The focus of this manuscript will be mainly on how specific types of cytologic and small biopsy samples can be triaged for appropriate ancillary molecular testing. This discussion will be in conjunction with basic pre-analytic requirements for ancillary tests, especially mutation analysis and FISH tests. The diversity of preparatory platforms given the various types of cytologic and small biopsy samples are summarized in Fig. 1. Although some background information regarding certain malignancies and ancillary testing techniques will be discussed, this discussion is not intended to be an in depth review of these aspects and only serve to provide appropriate contexts for the scope of this manuscript. Furthermore, specific topics such as sarcomas and lymphoproliferative disorders will be covered in other manuscripts within this special issue and will not be discussed here.

\section{Pre-analytical requirements for ancillary molecular and FISH testing}

Two general categories of polymerase chain reaction (PCR) based molecular testing methods are recognized: screening

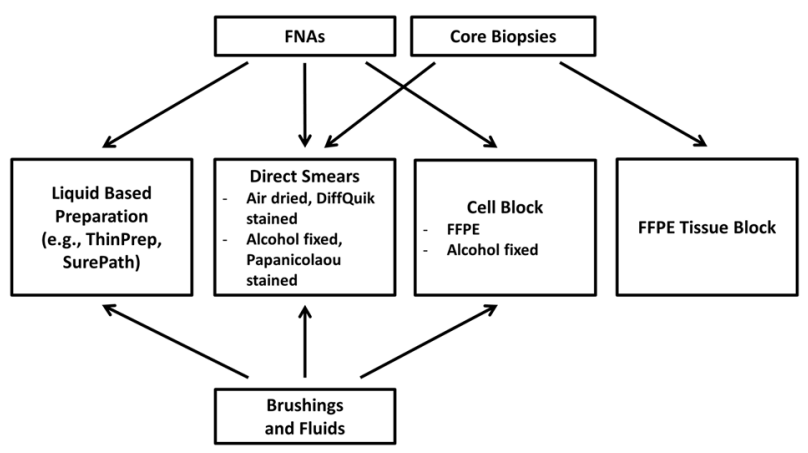

Fig. 1 Diversity of specimen and slide preparations for cytology and small biopsy specimens. FNA fine needle aspiration, FFPE formalin fixed paraffin embedded and targeted methods [4]. Sanger sequencing represents the prototypical example of the former and represents a method to screen for all mutations, known and novel variants, in a gene of interest. This is considered the gold standard and this technology is widely available. Nonetheless, this approach is relatively more labor intensive, leading to longer turnaround times, and more importantly, requires a relatively higher percent tumor cellularity sample. This equates to a relatively lower analytic sensitivity of this testing method. Within the purified nucleic acid sample being tested, the mutant allele must represent $15-20 \%$ of the allelic population overall (mutant plus wild type) in order for Sanger sequencing to detect the presence of the mutation. This equates to a cellular sample of approximately $30-40 \%$ tumor cells based on the assumption that activating oncogenic mutations are being tested (e.g., epidermal growth factor receptor [EGFR], RAS, or BRAF) and that the activating mutation is a heterozygous event without gene amplification, for the convenience of calculation. Thus, in this context, each tumor cell would harbor 50\% mutant allele and if DNA was purified from a pure tumor cell population (100\% percent tumor cellularity), the mutant allele frequency would be expected to be $50 \%$. If a sample harbored 50\% tumor cells and 50\% benign cells (e.g., lymphocytes), the latter population harboring two copies of the wild type allele, the mutant allele frequency would be $25 \%$. In a cellular sample composed of $10 \%$ tumor cells and $90 \%$ benign cells, the mutant allele frequency would be expected to be $5 \%$. In the first two scenarios, Sanger sequencing would be expected to detect the mutation in the gene being tested. However, in the final scenario, the mutant allele frequency would be too low and too diluted by the wild type allele that Sanger sequencing would fail to detect the mutation event. Because of the lower analytic sensitivity of Sanger sequencing, cytologic and small biopsy samples must often undergo a tumor cellularity enrichment technique prior to nucleic acid purification $[4,5]$. This is often accomplished by macrodissection or microdissection of tumor enriched areas from slide preparations such as cytologic slide preparations or unstained sections derived from FFPE tissue and cell block preparations [5, 6]. Due to the haphazard admixture of tumor and benign cellular elements, that is often encountered in cell block preparations especially, tumor cell enrichment from cell block sections can be particularly challenging.

As opposed to Sanger sequencing, alternative mutation detection approaches such as pyrosequencing and PCR based fragment analysis require a less pure tumor cellularity sample, typically in the range of $5-10 \%$ mutant allele (10-20\% tumor cells). Thus, these assays are associated with a higher analytic sensitivity. These techniques are typically considered targeted approaches to mutation analysis and are less time-consuming, which can lead to shorter 
turnaround times $[4,6]$. Nonetheless, the actual mutations that are detected are based on the design of the targeted assays; not every conceivable mutation, including rare mutations, would be detected. Recently, next generation sequencing (NGS) has emerged as a high throughput method for multiplexed gene analysis which enables for the detection of a diverse array of mutations [7-10] in multiple genes simultaneously. Therefore, NGS essentially represents a screening method of molecular testing. Furthermore, this testing approach benefits from higher analytic sensitivity; $5-10 \%$ mutant allele frequencies (10-20\% tumor cells) are typically required for mutation detection [6]. The Ion Torrent/PGM and Illumina/Mi Seq technologies represent prototypical examples [11].

Minimum cellular requirements for PCR based molecular assays depend on the nature of the testing platform and inherent quantitative and qualitative requirements inherent to the validated assays. Regardless, in light of the analytic sensitivities of various molecular testing methodologies, the pre-analytical slide evaluation is critical to identify tumor rich areas, especially if macrodissection or microdissection of tumor enriched areas is to be performed. Purification of nucleic acid from a high cellularity sample is not necessarily always desirable especially if the overall percent tumor cellularity is low since this could lead to a false negative mutation analysis result. Furthermore, failure of PCR based mutation analysis is especially more likely to be encountered in the setting of low cellularity and low percent tumor cellularity contexts [12-14]. Conversely, low cellularity/high percent tumor cellularity samples have been shown to be adequate for the detection of mutations. As an illustration, Allegrini and colleagues demonstrated the detection of EGFR mutations in lung cancer cytology samples utilizing very limited cellularity material (albeit high percent tumor cellularity) in the order of hundreds of cells [15]. Again, the lower limits of cells utilized for molecular assays depends on the design of the assay and quantitative aspects of assay validation. Of note, quantitative adequacy can be predicted based on the notion that 1 intact diploid cell yields approximately 6-7 pg of DNA [16, 17]. Therefore, a molecular test that requires $1 \mathrm{ng}$ or $10 \mathrm{ng}$ of DNA would equate to a total of approximately 143-167 or 1430-1670 intact cells, respectively. DNA amounts in these ranges can often be sufficient for molecular testing. For example, the DNA input required for the Ion Torrent NGS platform is typically in the order of $10 \mathrm{ng}$ [11]. The Illumina NGS platform, however, requires more DNA in the order of 30-270 ng [11]. Often times, it is not difficult to identify sufficient numbers of cells on cytologic slide preparations such as direct smears (Fig. 2).

The minimum cellular requirements for FISH assays are also assay dependent. The percent tumor cellularity
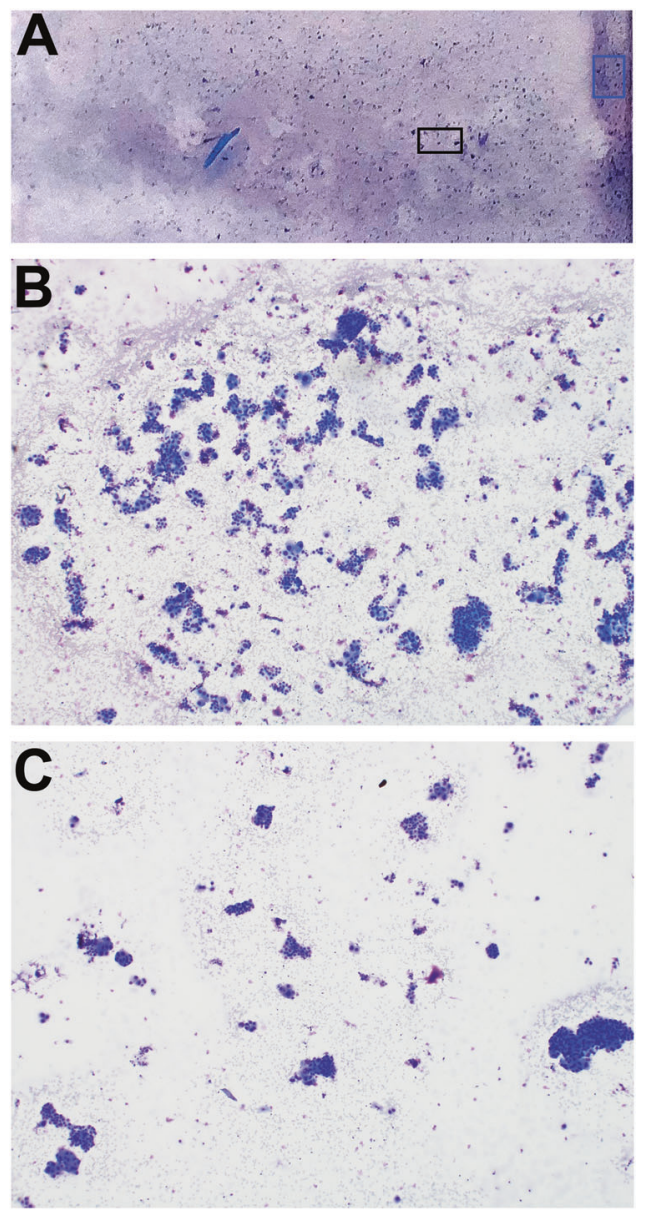

Fig. 2 Fine needle aspiration of metastatic lung adenocarcinoma. The Diff-Quik stained smear (a) appears hypocellular; the area in the blue and black boxes on the smear is shown in (b) and (c), respectively. Greater than 500 tumor cells are seen in (b). Greater than 200 tumor cells are seen in (c). Overall, on the entire smear, the cellularity was in excess of 10000 cells with at least $80 \%$ of these cells representing tumor cells. This smear would be expected to contain more than adequate cellularity for molecular testing

is less of a concern with the caveat that tumor nuclei on the slide can be easily distinguished from the nuclei of background benign cells under fluorescence microscopy based on nuclear size, morphology, and chromatin texture. The minimum number of tumor nuclei needed to be examined varies depending on the FISH assay. However, low tumor cellularity samples can be utilized for FISH analysis. As an illustration, FISH testing for ALK rearrangements in non-small cell lung carcinoma typically involves the examination of only 50 to 100 tumor nuclei $[18,19]$. In deserves mention that whole nuclei can be examined on cytologic slide preparations such as direct smears, cytospin slides, touch imprints, and liquid based cytologic slide preparations in contrast to sections obtained from formalin fixed paraffin embedded (FFPE) blocks. Thus, FISH analysis of the latter is more susceptible to nuclear truncation artifact. 
Fig. 3 Two cases of metastatic adenocarcinoma in exfoliative effusion cytology specimens. Representative

photomicrographs are shown for Papanicolaou stained ThinPrep slides (a, c) and corresponding H\&E stained cell block sections (b, d). Effusion specimens can be enriched in tumor cells with minimal background benign cellular elements $(\mathbf{a}, \mathbf{b})$ or be diluted by abundant background benign cellular elements including mesothelial cells and inflammatory cells $(\mathbf{c}, \mathbf{d})$. In (c) and (d), the arrows point to the malignant glandular cell clusters

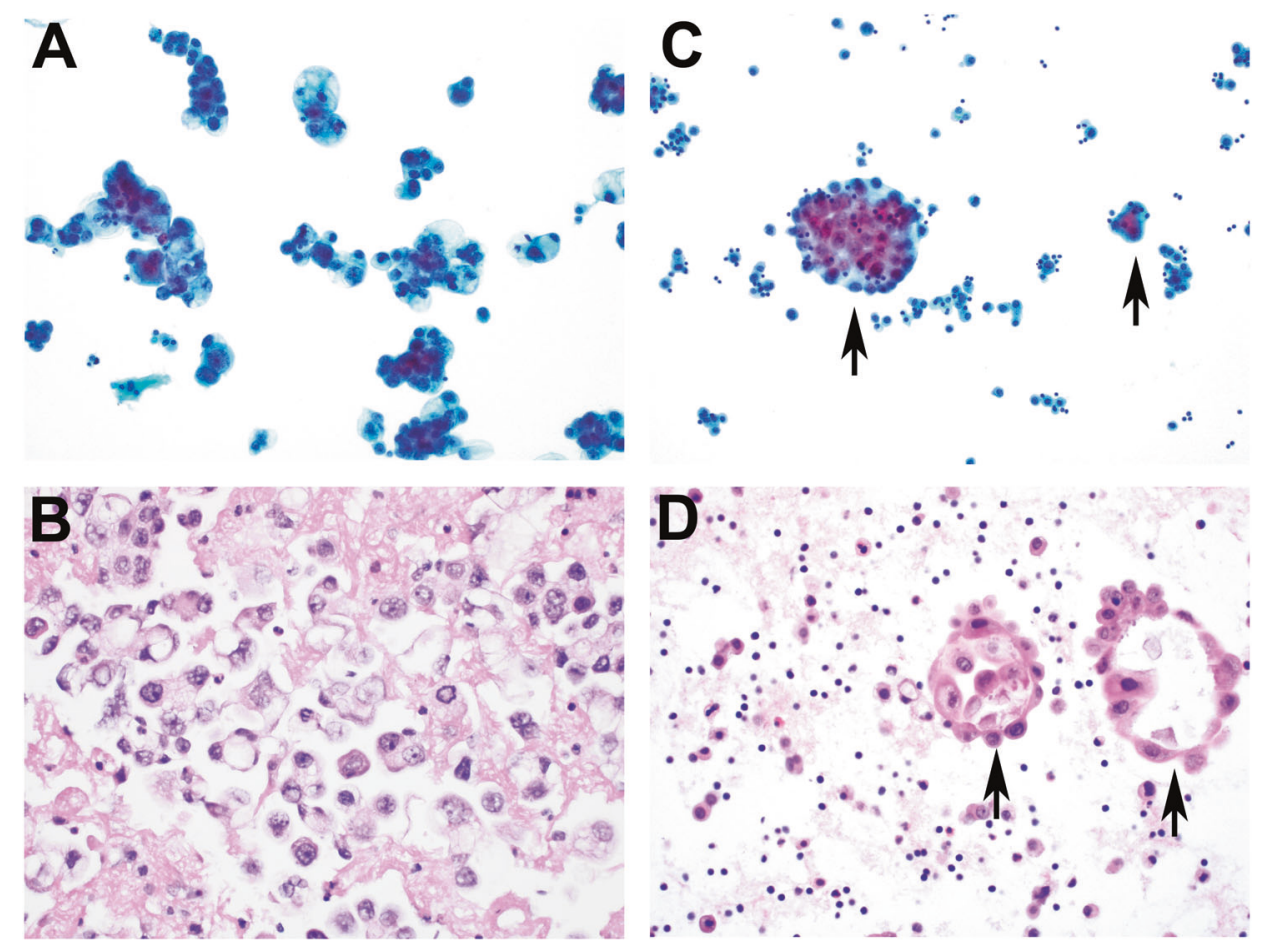

\section{Cytologic samples, small biopies, and preparatory platforms}

Cytologic specimens are generally classified in one of two broad categories: exfoliative and aspiration samples. Examples of exfoliative cytology specimens include cervicovaginal Pap specimens, urinary tract cytologic samples, bronchial brushings, bronchial washings, pancreatobiliary brushings, and effusion specimens. With regards to effusion specimens, they have the potential to provide high volume samples with abundant tumor cells (Fig. 3a, b). Nonetheless, it is not uncommon to encounter a situation in which the tumor cell population is admixed with abundant background benign cellular elements such as mesothelial cells and inflammatory cells (Fig. 3c, d). This has implications for mutation analysis as assays associated with a lower analytic sensitivity will have a higher likelihood for producing a false negative result in the latter setting. In this regard, a targeted mutation assay with higher analytic sensitivity, thereby requiring a lower percentage of tumor cells, would have a greater chance of success in detecting a mutation, if present in the tumor cells.

Aspiration cytology specimens mainly present in the form of FNAs and a variety of anatomic sites are amenable for sampling utilizing this rapid, inexpensive, minimally invasive method. Depending on the needle pass, a higher percentage tumor cell population can be obtained; nonetheless, a finite volume of specimen is acquired using this method. Rapid on-site evaluation (ROSE) can be employed for FNA procedures which allows for a cytotechnologist and/or pathologist to be present during the procedure. This allows for real-time determination of specimen adequacy for diagnosis and anticipated ancillary tests. ROSE represents an opportunity for close communication between the interventional proceduralist and pathology staff regarding the relevant patient-centered clinical question(s), preliminary diagnoses or differential diagnostic considerations, and any relevant ancillary testing that would be necessary $[2,5,20]$. This would have direct implications on how much additional specimen is procured and how the collected specimen material is triaged enabling appropriate testing that would be performed using these finite samples.

Needles of greater diameter enable for acquisition of core biopsy samples [21], which represent thin, cylindrical tissue biopsy samples of tumors. When touch imprint smears are prepared prior to the biopsy sample being harvested into fixative, such as neutral buffered formalin, a preliminary assessment of the these biopsies can be achieved. Evaluation of touch imprint smears can be performed at the time of the procedure during ROSE or in the interim time period between the conclusion of the procedure and gross processing of the biopsy in the laboratory. If smears are prepared, it is important for the tissue to be handled gently to ensure minimal adverse effect on the integrity of the tissue to be processed. Based on the clinical context and the preliminary assessment of the smears, immunohistochemical stains and extra unstained sections can be ordered upfront such that the desired number of unstained sections can be prepared at the time of initial microtomy. This proactive approach helps to minimize refacing of the block 
that would occur if repeat microtomy were to take place. This approach is commonly employed at this author's institution for select core biopsies destined to be submitted to the cytopathology laboratory. Upon receipt of alcohol fixed, unstained touch imprint smears and core biopsy tissue, the smears are Papanicolaou stained immediately and reviewed shortly thereafter by the cytopathologist. The cytopathologist, therefore, is in the position of ordering relevant immunostains and extra unstained sections upfront. Furthermore, in the appropriate clinical contexts, the cytopathologist can guide grossing of the tissue. As an illustration, in the setting of core biopsies of bone lesions, the cytopathologist can provide instructions to avoid decalcification agents as these agents are detrimental to the tissue for molecular testing purposes. Specifically, a biopsy sample taken from a bone lesion involved by metastatic carcinoma could be split such that softer tissue fragments avoid decalcification and the harder tissue fragments are decalcified allowing for effective sectioning of those tissue sections. In this context, for instance, the former be prioritized for PCR based molecular testing and the latter utilized for immunohistochemistry and FISH testing. After tissue processing, $H \& E$ sections along with the requisite number of unstained sections would be prepared upfront during initial microtomy. At the time of histologic review of the tissue sections, the H\&E stained slide along with first batch of immunohistochemical stains are able to be reviewed simultaneously providing the opportunity for a final, detailed diagnosis to be rendered on the following day after the procedure. The upfront preparation of extra additional unstained sections, especially for already requested or anticipated ancillary testing, is particularly useful for enabling timely, efficient triage of these patient assets for appropriate molecular tests once the diagnosis has been rendered.

FNAs and core biopsies are often complementary. To opine that one modality is universally better than the other is difficult as, on a case by case basis, one modality may yield a better specimen than the other and vice versa. Furthermore, the quality of the procured sample highly depends on the skill of the operator in performing the procedure; this aspect cannot be overemphasized [22]. FNA has the potential to sample a wider area of the target lesion within a given needle pass as the angle and trajectory can be altered during each successive incursion and excursion of the needle. FNA can also allow for acquisition of tumor cells with lower contamination by stromal tissue. The core biopsy can be associated with a larger volume of intact tissue fragments; however, tumor and stroma are often sampled together. This has implications for molecular testing given analytic sensitivity considerations. In addition, FNAs are considered to be safer as core biopsies can be associated with a higher rate of complications such as bleeding, especially when sampling highly vascular lesions/ organs, and damage to nerves, in the setting of lesions and organs that are in the vicinity of major nerve branches [22]. FNAs offer more flexibility in terms of cytologic specimen processing given the diversity of cytopreparatory platforms such as direct smears, liquid based preparations, and cell blocks whereas core biopsies are often destined for FFPE block preparations (Fig. 1). For FNAs, this is associated with increased efforts for molecular test development as this requires additional validation of non-FFPE platforms along with FFPE preparations [6]. Direct smears can be prepared rapidly with minimal cost expense. Per needle pass, the option also exists to distribute cellular material over multiple smears which allows for greater flexibility in terms of triaging direct smears for various ancillary studies (Fig. 4). With regards to direct smears and liquid based cytologic slide preparations, the tumor cells are not exposed to formalin and yield higher quality nucleic acid for molecular tests. Air-dried, Diff Quik stained smears and alcohol-fixed Papanicolaou stained cytologic slides, including smears and liquid based preparations, have all been shown to be effective for molecular and FISH testing [16, 23-25]. Nonetheless, the use of these slide preparations for these purposes often requires additional validation since traditionally, FFPE blocks prepared from tissue samples, core biopsies, and cell blocks are most often used and validated for ancillary immunohistochemical, molecular, and FISH assays. Therefore, for convenience, cell blocks have traditionally represented the main source of cytologic specimen material for these tests. Nonetheless, in the setting of FNAs, sole reliance on cell blocks for ancillary testing can lead to situations in which insufficient material is available for

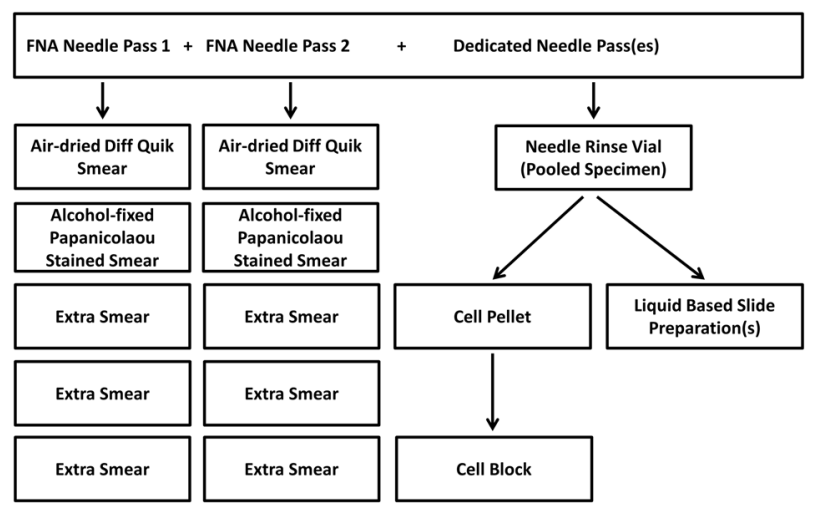

Fig. 4 Typical slide preparation procedures for fine needle aspiration (FNA) specimens. If smears are prepared from a needle pass, a pair of smears-air-dried, Diff-Quik stained and alcohol-fixed, Papanicolaou stained smears-are commonly prepared; however, cellular material can often be distributed over more than two smears per needle pass. This can result in extra smears being available for ancillary studies, if needed. Needle rinses are commonly performed for FNAs, including dedicated needle rinses, and the resulting pooled cellular suspension can be utilized for the preparation of a cell block and/or liquid based slide preparation(s) 
this purpose [2]. It is recognized that adequate cellularity of cell blocks cannot be guaranteed at the time of the procedure, despite the utilization of ROSE, even if dedicated passes for the needle rinse are collected. This in contrast to direct smear preparations, especially air-dried Diff Quik stained smears, as the cellularity on these slides can be assessed under the microscope, shortly after cellular procurement, during the procedure while the patient is still accessible. Furthermore, a pooled needle rinse from all FNA passes is commonly utilized for centrifugation and cell block preparation. This presents one additional drawback of cell blocks due to the potential for tumor cell dilution by benign cellular elements such as lymphocytes $[2,5,20]$. To illustrate this point, this would occur in the context of differential sampling of tumor cell rich and tumor cell poor areas of a lymph node partially replaced by metastatic cancer (Fig. 5). This could lead to false negative results if a molecular test with lower analytic sensitivity is utilized.

Overall, the specimen type and clinical context will determine how cytologic and small biopsy samples and slide preparations are prioritized for pathologic evaluation

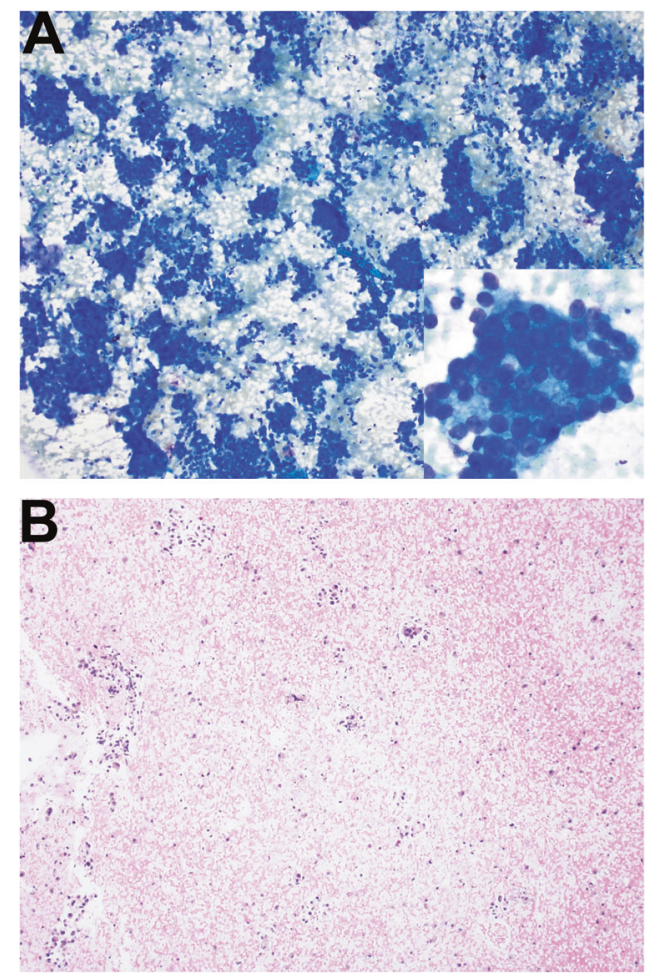

Fig. 5 Fine needle aspiration of metastatic lung adenocarcinoma. DiffQuik stained smears (a) prepared from a subset of needle passes yielded essentially a pure tumor cellularity sample composed of sheets and three-dimensional acinar epithelial clusters (inset). However, other needle passes yielded abundant lymphocytes (not shown) and the pooled needle risnse was utilized to prepare a cell block. The H\&E stained cell block section (b) revealed an admixture of lymphocytes and tumor cells and ancillary molecular testing. Select specific situations are discussed next, given the previous discussion of preanalytic specimen requirements of various molecular testing methodologies, such that these concepts can be illustrated with regards to specimen handling and triage procedures. Molecular testing of non-small cell lung carcinoma (NSCLC) and metastatic melanoma has diversified and represent salient examples of how molecular testing has been intimately associated with cytologic and small biopsy samples; this will be discussed in the next section to illustrate the application of the aforementioned principles.

\section{Clinical applications}

Within the past decade, there has been dramatic growth with respect to molecular testing of NSCLC and metastatic melanoma samples [26, 27]. A significant proportion of patients with NSCLC and melanoma are diagnosed with advanced stage disease. In this context, cytologic samples and small biopsies are increasingly being relied upon to establish a diagnosis and perform a multitude of ancillary prognostic and theranostic tests [1, 3, 28, 29]. Initially, for NSCLC, molecular testing centered on interrogating EGFR and KRAS mutation status. Currently, however, the list of clinically relevant genetic markers being tested has increased. Now it is not uncommon to encounter orders for ancillary testing for a variety of genes such as EGFR, ALK, ROS1, HER2, KRAS, BRAF, and RET along with PD-L1 expression status in NSCLC samples. Likewise, for metastatic melanoma specimens, BRAF mutation analysis initially represented the mainstay of molecular testing. However, mutation analysis for additional genes, such as c-kit, GNAQ, GNA11, c-kit, and NRAS, are being increasingly requested. These lists will likely continue to grow based on further advances in the discovery of novel biomarkers underlying the pathogenesis of lung cancer and metastatic melanoma.

This has implications for specimen processing and triage procedures given the differential nature of the assays used to assess the status of these genes and biomarkers. Mutation testing for oncogenes such as EGFR, BRAF, c-kit and RAS is typically performed using PCR based methods. ALK, ROS1, and RET rearrangement testing is often accomplished using FISH; however, immunohistochemistry has emerged as a screening tool for assessing for ALK and ROS1 rearrangements [19, 30-33]. High throughput analysis of multiple genes can be achieved also via NGS which can increase efficiency of molecular testing and can have the potential for more efficacious use of limited cytologic and small biopsy material [9, 10, 34-38]. PD-L1 status is assessed by immunohistochemistry and it should be 
noted that although this has not been validated for cytology specimens, studies have emerged demonstrating that comparable results can be achieved, with respect to histologic specimens [39]. For cytologic samples, cell blocks are traditionally utilized for immunohistochemistry based testing, molecular testing, and FISH analysis. Nonetheless, cytologic slide preparations, such as direct smears and liquid based preparations, have been shown to be an effective source of cellular material for these ancillary tests and have been validated by an increasing number of laboratories. Depending on the pathologist's access to various ancillary testing methodologies validated on various platforms, prioritization of specimen preparation methodologies and triage of these patient slides and blocks for various molecular tests can be determined given the cellularity characteristics of these sample preparations.

Figure 5 illustrates a case of an FNA sampling of a mediastinal lymph node involved by NSCLC, specifically, an adenocarcinoma (Fig. 5). While some passes preferentially sampled a tumor rich area, other passes sampled tumor poor areas enriched with lymphocytes (not shown). Dedicated FNA passes were performed for the pooled needle rinse and the cell block prepared from this yielded an admixture of lymphocytes and tumor cells with an overall percent tumor cellularity of less than $10 \%$. Given the analytic sensitivity issues discussed previously, the effectiveness of the cell block preparation for PCR based molecular testing and NGS testing would be expected to be limited with a significant likelihood of a false negative result. However, the existence of direct smears enriched with tumor cells is fortunate in this scenario and would be expected to be an excellent source of material for these testing methodologies. The cell block preparation could be better reserved for immunohistochemical and FISH based testing methodologies for which the low percent tumor cellularity is less problematic of an issue. In this case, the cell block was utilized for immunohistochemical verification of the diagnosis of lung adenocarcinoma; a TTF1-positive and p40-negative immunophenotype was observed (not shown). At this author's institution, NGS is commonly utilized to test for somatic alterations in genes including BRAF, EGFR, ERBB2, HRAS, KRAS, MET, and NRAS along with gene rearrangements involving ALK, RET, ROS1, and NTRK1. NGS was performed utilizing tumor cells scraped from a direct smear and although the test was resulted as negative, there was confidence that this was not a false negative result considering that essentially a pure tumor population was tested.

Figure 6 illustrates a case of a core biopsy obtained from a liver lesion in a patient with a history of ocular melanoma. Touch imprint smears were prepared utilizing the tissue cores during the procedure. The core biopsies along with the unstained smears were submitted to the cytopathology
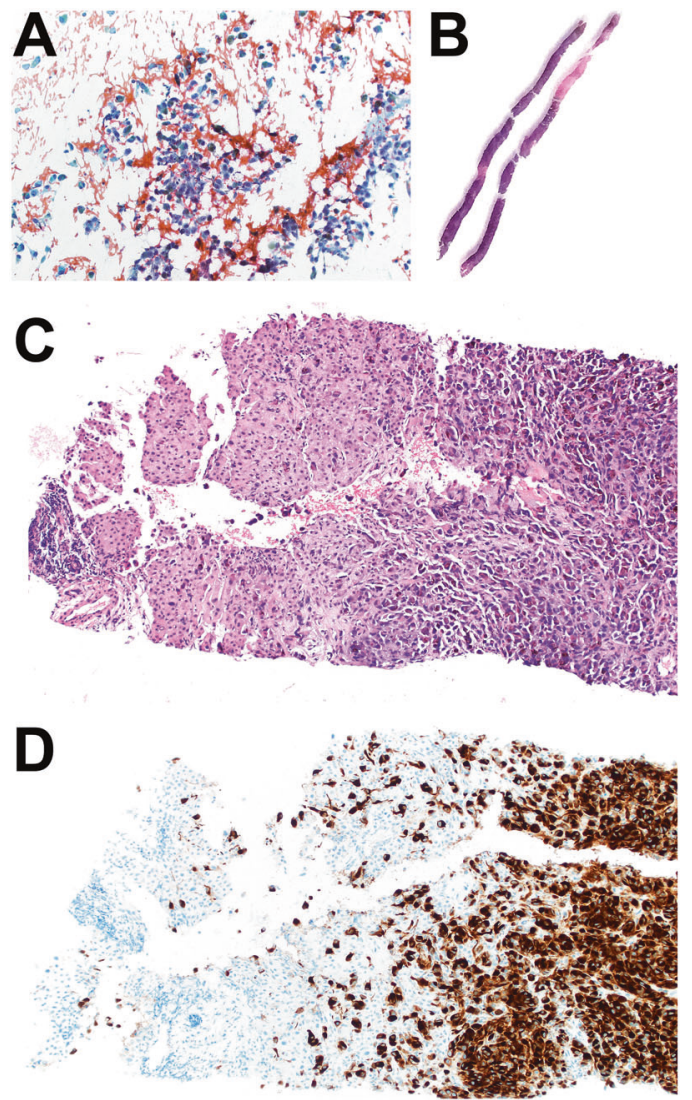

Fig. 6 Liver core biopsy with touch imprint smear in a case of metastatic melanoma. Representative area of a Papanicolaou stained touch imprint smear (a) showing a neoplastic population of epithelioid cells. The core biopsy (b) demonstrates the presence of metastatic melanoma involving liver parenchyma (c); non-neoplastic liver parenchyma is seen on the left aspect of the image whereas the metastatic tumor is seen on the right aspect of the image (c). Immunohistochemistry for Melan A highlights the metastatic tumor cells confirming the diagnosis of metastatic melanoma (d)

laboratory for processing. The smears were rapidly stained within the cytopathology laboratory for a preliminary assessment. In this situation, the clinical impression favored metastatic melanoma; if this was confirmed, NGS based molecular testing was desired. At this author's institution, a melanoma targeted gene panel is utilized for this purpose and evaluates for somatic mutations within BRAF, GNAQ, GNA11, KIT, and NRAS. Similar to the lung cancer NGS panel discussed above, this test has been validated for use in FFPE sections as well as cytologic slide preparations; at least 5000 total nucleated cells and at least 20\% tumor cells are required for both NGS assays. The touch imprint smears demonstrated the presence of a neoplastic epithelioid cell population and melanin pigment was not readily appreciable (Fig. 6). Since the patient's original melanoma pathology report indicated immunoreactivity for Melan A, immunohistochemistry for Melan A was ordered along with an H\&E stained slides and multiple 
unstained sections upfront at the time of initial microtomy. On the following day, H\&E sections from the core biopsies were ready for evaluation (Fig. 6b) along with the Melan A immunostain and extra unstained slides. Melanin pigment was better appreciated in the histologic sections (Fig. 6c) and Melan A was diffusely positive in the neoplastic cells of interest (Fig. 6d) confirming the diagnosis of metastatic melanoma. The unstained slides were immediately triaged for NGS testing. The areas on the unstained slides that corresponded to the tumor enriched areas on the H\&E slide were scraped, taking care to avoid the areas corresponding to non-neoplastic liver parenchyma, and harvested for nucleic acid extraction. Ultimately, a Q209L mutation in GNA11 was detected.

With the aforementioned examples, two additional points deserve mention. First, these examples highlight the recognition that a minimal panel of immunostains may be desired to help preserve tissue in the FFPE block for molecular studies. Second, the presence of a smear enriched in tumor cells provides an additional option for cellular material to be utilized for molecular testing. This especially applies in the event that a tumor cell enriched area on the tissue sections, associated with a percentage of tumor cells exceeding the limit of detection of the assay, cannot be identified for microdissection and subsequent nucleic acid extraction.

\section{Concluding remarks}

The menu of ancillary testing, including mutation analysis, rearrangement analysis, and FISH, applied to finite cytologic and small biopsy samples have continued to diversify. Pathologists are tasked with leveraging these limited patient samples not only for rendering an accurate diagnosis, which frequently involves use of immunohistochemical stains, but also for ancillary molecular tests that contribute to the diagnosis and/or provide downstream prognostic and theranostic information. By understanding the specimen testing requirements for these various assays, the pathologist can be in a powerful position to help optimally triage various sample preparations for successful ancillary testing of these finite specimens.

\section{Compliance with ethical standards}

Conflict of interest The author declares that he has no conflict of interest.

\section{References}

1. Clark DP. Seize the opportunity: underutilization of fine-needle aspiration biopsy to inform targeted cancer therapy decisions. Cancer. 2009;117:289-97.
2. Knoepp SM, Roh MH. Ancillary techniques on direct-smear aspirate slides: a significant evolution for cytopathology techniques. Cancer Cytopathol. 2013;121:120-8.

3. Krishnamurthy S. Applications of molecular techniques to fineneedle aspiration biopsy. Cancer. 2007;111:106-22.

4. Ellison G, Zhu G, Moulis A, et al. EGFR mutation testing in lung cancer: a review of available methods and their use for analysis of tumour tissue and cytology samples. J Clin Pathol. 2013;66:79-89.

5. Roh MH. The utilization of cytologic fine-needle aspirates of lung cancer for molecular diagnostic testing. J Pathol Transl Med. 2015;49:300-9.

6. Jain D, Roy-Chowdhuri S. Molecular pathology of lung cancer cytology specimens: a concise review. Arch Pathol Lab Med 2018;142:1127-33.

7. Lozano MD, Echeveste JI, Abengozar M, et al. Cytology Smears in the Era of Molecular Biomarkers in Non-Small Cell Lung Cancer: Doing More With Less. Arch Pathol Lab Med. 2018;142: 291-8.

8. Nikiforov YE, Carty SE, Chiosea SI, et al. Impact of the MultiGene ThyroSeq Next-Generation Sequencing Assay on Cancer Diagnosis in Thyroid Nodules with Atypia of Undetermined Significance/Follicular Lesion of Undetermined Significance Cytology. Thyroid. 2015;25:1217-23.

9. Roy-Chowdhuri S, Goswami RS, Chen H, et al. Factors affecting the success of next-generation sequencing in cytology specimens. Cancer Cytopathol. 2015;123:659-68.

10. Treece AL, Montgomery ND, Patel NM, et al. FNA smears as a potential source of DNA for targeted next-generation sequencing of lung adenocarcinomas. Cancer Cytopathol. 2016; $124: 406-14$

11. Vigliar E, Malapelle U, de Luca C, et al. Challenges and opportunities of next-generation sequencing: a cytopathologist's perspective. Cytopathology. 2015;26:271-83.

12. Betz BL, Roh MH, Weigelin $\mathrm{HC}$, et al. The application of molecular diagnostic studies interrogating EGFR and KRAS mutations to stained cytologic smears of lung carcinoma. Am J Clin Pathol. 2011;136:564-71.

13. Billah S, Stewart J, Staerkel G, et al. EGFR and KRAS mutations in lung carcinoma: molecular testing by using cytology specimens. Cancer Cytopathol. 2011;119:111-7.

14. Hookim K, Roh MH, Willman J, et al. Application of immunocytochemistry and BRAF mutational analysis to direct smears of metastatic melanoma. Cancer Cytopathol. 2012;120:52-61.

15. Allegrini S, Antona J, Mezzapelle R, et al. Epidermal growth factor receptor gene analysis with a highly sensitive molecular assay in routine cytologic specimens of lung adenocarcinoma. Am J Clin Pathol. 2012;138:377-81.

16. Dejmek A, Zendehrokh N, Tomaszewska M, et al. Preparation of DNA from cytological material: effects of fixation, staining, and mounting medium on DNA yield and quality. Cancer Cytopathol. 2013;121:344-53.

17. Ranek L. Cytophotometric studies of the DNA, nucleic acid and protein content of human liver cell nuclei. Acta Cytol. 1976;20:151-7.

18. Betz BL, Dixon CA, Weigelin $\mathrm{HC}$, et al. The use of stained cytologic direct smears for ALK gene rearrangement analysis of lung adenocarcinoma. Cancer Cytopathol. 2013;121: 489-99.

19. Minca EC, Lanigan CP, Reynolds JP, et al. ALK status testing in non-small-cell lung carcinoma by FISH on ThinPrep slides with cytology material. J Thorac Oncol. 2014;9:464-8.

20. Roh MH. Triage of cytologic direct smears for ancillary studies: a case-based illustration and review. Arch Pathol Lab Med. 2013;137:1185-90. 
21. VanderLaan PA. Fine-needle aspiration and core needle biopsy: an update on 2 common minimally invasive tissue sampling modalities. Cancer Cytopathol. 2016;124:862-70.

22. van Zante A, Ljung BM. Fine-needle aspiration versus core needle biopsy: reconsidering the evidence of superiority. Cancer Cytopathol. 2016;124:853-6.

23. Bellevicine C, Malapelle U, de Luca C, et al. EGFR analysis: current evidence and future directions. Diagn Cytopathol. 2014; 42:984-92.

24. da Cunha Santos G, Saieg MA, Geddie W, et al. EGFR gene status in cytological samples of nonsmall cell lung carcinoma: controversies and opportunities. Cancer Cytopathol. 2011;119: 80-91.

25. Killian JK, Walker RL, Suuriniemi M, et al. Archival fine-needle aspiration cytopathology (FNAC) samples: untapped resource for clinical molecular profiling. J Mol Diagn. 2010;12:739-45.

26. Lindeman NI, Cagle PT, Aisner DL, et al. Updated molecular testing guideline for the selection of lung cancer patients for treatment with targeted tyrosine kinase inhibitors: guideline from the College of American Pathologists, the International Association for the Study of Lung Cancer, and the Association for Molecular Pathology. J Thorac Oncol. 2018;13:323-58.

27. Woodman SE, Lazar AJ, Aldape KD, et al. New strategies in melanoma: molecular testing in advanced disease. Clin Cancer Res. 2012;18:1195-1200.

28. Aisner DL, Sams SB. The role of cytology specimens in molecular testing of solid tumors: techniques, limitations, and opportunities. Diagn Cytopathol. 2012;40:511-24.

29. Rekhtman N, Roy-Chowdhuri S. Cytology specimens: a goldmine for molecular testing. Arch Pathol Lab Med. 2016;140:1189-90.

30. Bubendorf L, Buttner R, Al-Dayel F, et al. Testing for ROS1 in non-small cell lung cancer: a review with recommendations. Virchows Arch. 2016;469:489-503.
31. Savic S, Bode B, Diebold J, et al. Detection of ALK-positive non-small-cell lung cancers on cytological specimens: high accuracy of immunocytochemistry with the 5A4 clone. J Thorac Oncol. 2013;8:1004-11.

32. Sholl LM, Sun H, Butaney M, et al. ROS1 immunohistochemistry for detection of ROS1-rearranged lung adenocarcinomas. Am J Surg Pathol. 2013;37:1441-9.

33. Vlajnic T, Savic S, Barascud A, et al. Detection of ROS1-positive non-small cell lung cancer on cytological specimens using immunocytochemistry. Cancer Cytopathol. 2018;126:421-9.

34. Hwang DH, Garcia EP, Ducar MD, et al. Next-generation sequencing of cytologic preparations: An analysis of quality metrics. Cancer Cytopathol. 2017;125:786-94.

35. Kanagal-Shamanna R, Portier BP, Singh RR, et al. Nextgeneration sequencing-based multi-gene mutation profiling of solid tumors using fine needle aspiration samples: promises and challenges for routine clinical diagnostics. Mod Pathol. 2014;27: 314-27.

36. Karnes HE, Duncavage EJ, Bernadt CT. Targeted next-generation sequencing using fine-needle aspirates from adenocarcinomas of the lung. Cancer Cytopathol. 2014;122:104-13.

37. Reynolds JP, Zhou Y, Jakubowski MA, et al. Next-generation sequencing of liquid-based cytology non-small cell lung cancer samples. Cancer Cytopathol. 2017;125:178-87.

38. Young $\mathrm{G}$, Wang $\mathrm{K}$, $\mathrm{He} \mathrm{J}$, et al. Clinical next-generation sequencing successfully applied to fine-needle aspirations of pulmonary and pancreatic neoplasms. Cancer Cytopathol. 2013; 121:688-94.

39. Torous VF, Rangachari D, Gallant BP, et al. PD-L1 testing using the clone $22 \mathrm{C} 3$ pharmDx kit for selection of patients with nonsmall cell lung cancer to receive immune checkpoint inhibitor therapy: are cytology cell blocks a viable option? J Am Soc Cytopathol. 2018;7:133-41. 